\title{
An Autopsied Case of an Apparent Pontine Branch Atheromatous Disease
}

\author{
Shinsui Tatsumi Toru Yamamoto \\ Department of Neurology, Osaka Saiseikai Nakatsu Hospital, Osaka, Japan
}

Dear Sir,

Based on 3 autopsied cases with pontine infarcts $[1,2]$, the 'branch atheromatous disease' was proposed by Caplan [3] in 1989 as a new distinct category of cerebral infarction caused by a localized atheromatous lesion at the mouth of the branch from the major artery's branch. Although there has been no histopathological report since then, some types of infarction are believed to fit into that category in clinical practice. Here we present the case with MR images compatible with 'branch atheromatous disease', but the histopathological findings were those of a large artery atherothrombotic infarct.

A 79-year-old man with risk factors of diabetes mellitus, hyperlipidemia and smoking was admitted to our hospital because of complete left-sided hemiplegia in October 2006. Brain MRI revealed an acute right-sided pontine infarct reaching its ventral surface (in the territory of the paramedian, and short and long circumferential arteries), along with multiple old lacunes in the bilateral basal ganglia. The basilar artery appeared normal on MRI/ MRA (fig. 1A). The patient remained normotensive without medication. Nine months later, he died of pneumonia. The main findings of general pathology were pneumonia and mild diabetic nephropathy. Neuropathological findings included an old cavitary infarction of the right pontine base (fig. 1B), multiple lacunar infarcts in the bilateral basal ganglia, and slight senile changes. At the brain base, severe atherosclerosis was observed. We separated the basilar artery from the brain, and evaluated it posteriorly. One right-sided branch at the level of the pontine infarct lost transparency at its most proximal segment (fig. 1B). Serial $4-\mu \mathrm{m}$ thick cross sections of the basilar artery including that branch were made for about $5 \mathrm{~mm}$ of length.

Organized thrombosis with recanalization was observed at the origin of that branch, where numerous macrophages were infiltrated within the fibrous atheroma (fig. 1C). Throughout the serial sections, the parental basilar artery was markedly atherosclerotic without thrombus formation (fig. 1C). There were additional 6 branches from the basilar artery in the sections. One left-sided branch at the same level of the aforementioned right-sided branch was stenosed by macrophage plaque at its origin without an associated infarction. The other 5 were running through the thick atheromatous basilar wall without overt macrophage plaques or stenosis (fig. 1C).

There was a discrepancy of MR images and pathology in this case. MRI findings of the basilar artery appeared normal probably because of arterial pressure and ectatic effect. However, its actual pathology was definitely moderate to severe atherosclerosis.

In general, a branching site of the artery is prone to atherothrombosis [4], and this may be true with the basilar artery. In our case, the MR images showed a pontine infarct reaching to its ventral surface, which is compatible with the 'branch atheromatous disease' [3]. However, the histology was junctional atherothrombosis on the background of the severely atherosclerotic basilar artery. So the lack of atheroma in the basilar artery that Caplan stressed is not a requisite for this type of infarction.
References

1 Fisher CM, Caplan LR: Basilar artery branch occlusion: a case of pontine infarction. Neurology 1971;21:900-905.

2 Fisher CM: Bilateral occlusion of basilar artery branches. J Neurol Neurosurg Psychiatry 1977;40:1182-1189.
3 Caplan LR: Intracranial branch atheromatous disease: a neglected, understudied, and underused concept. Neurology 1989;39: 1246-1250.

4 Kalimo H, Kaste M, Haltia M: Vascular diseases; in Graham DI, Lantos PL (eds): Greenfield's Neuropathology, ed 7. London, Arnold, 2002, vol I, pp 288-291.

\section{KARGER}

Fax +4161306 1234 E-Mail karger@karger.ch www.karger.com
Shinsui Tatsumi, MD

Department of Neurology, Osaka Saiseikai Nakatsu Hospital

2-10-39 Shibata, Kita-ku, Osaka 530-0012 (Japan)

Tel. +81663720333 , Fax +81663720339

E-Mailshinsuit@nakatsu.saiseikai.or.jp 

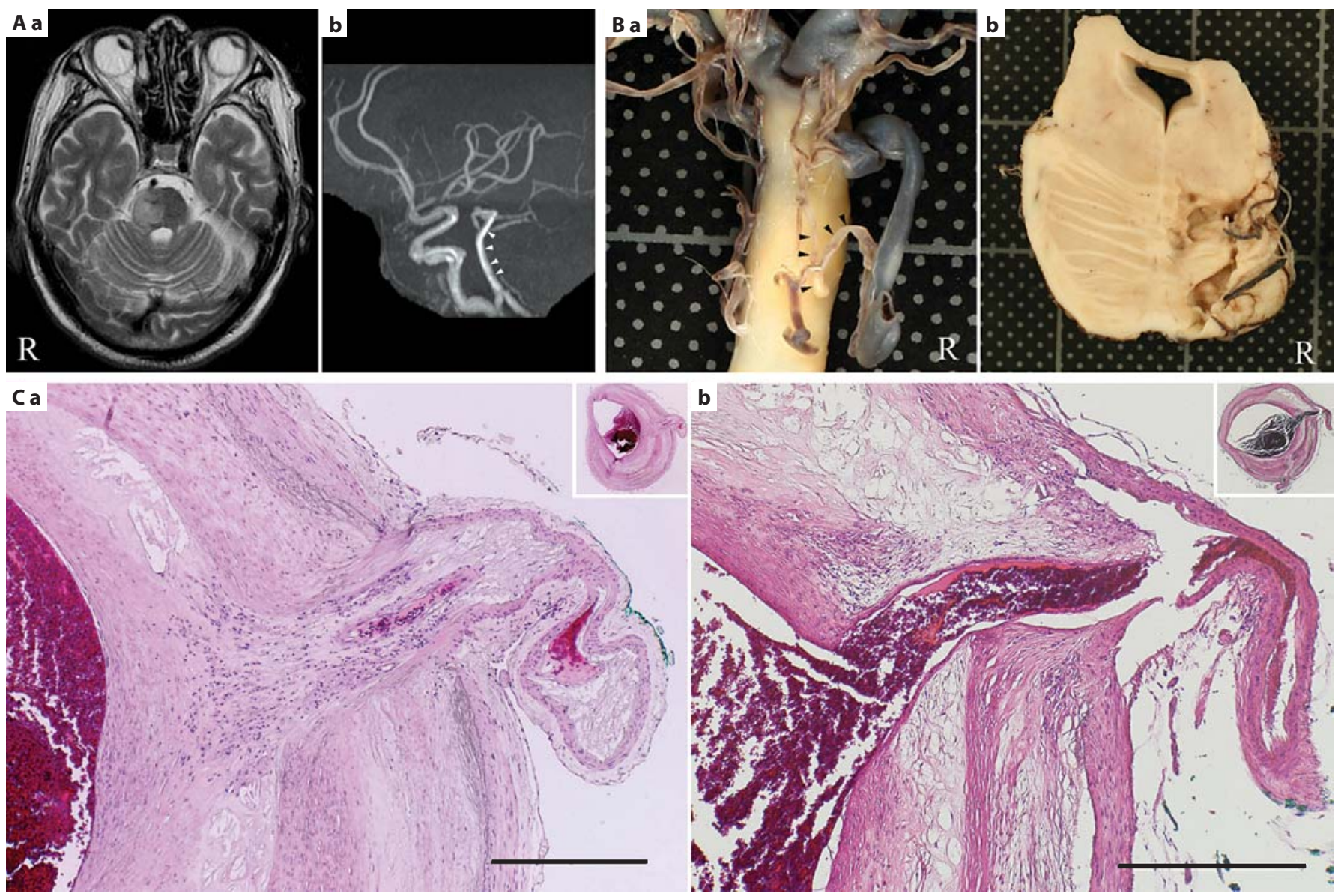

Fig. 1. MRI and neuropathology. $\mathbf{A} \mathrm{T}_{2}$-weighted image (a) and MRA (b) showing a right-sided hemipontine infarct and unremarkable basilar artery (arrowheads). B The posterior view of the basilar artery showing that one right-sided branch (arrowheads) lost transparency at its origin (a, two different views). This branch bifurcates just after its origin. A cavitary infarction involves near- ly the whole right pontine base (b). C Marked atherosclerosis of the basilar artery (inset) and the branch with organized thrombosis and macrophage infiltration at its origin (HE) (a). Another branch without stenosis running through the atheromatous basilar wall (70 slices caudal to C (a); HE) (b). Bar $=500 \mu \mathrm{m}$. 